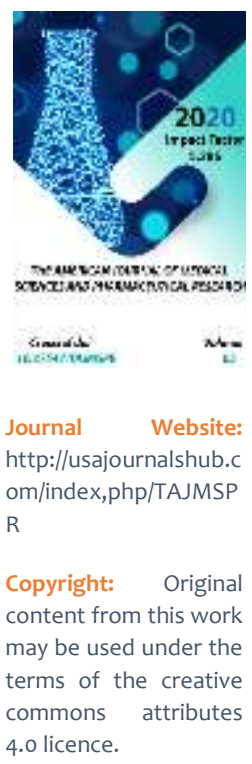

\title{
Primary Diagnosis Of Acquired Thrombocytopathy In Family Polyclinics
}

\author{
Dadajanov Utkir Dadajanovich \\ Associate Professor, Head Of The Department Department Of Hematology, Candidate Of \\ Medical Sciences, Samarkand State Institute, Uzbekistan
}

Abdiev Kattabek Makhmatovich

Associate Professor Of The Department Of Hematology, Candidate Of Medical Sciences, Samarkand State Institute, Uzbekistan

\section{ABSTRACT}

The article is devoted to general issues of classification and differential diagnosis of thrombocytopathy. Features of pathogenesis, clinical course and diagnosis of certain rare inherited thrombocytopathies are highlighted. Particular attention is paid to the formation of the molecular basis of these diseases. The basic genetic mutations associated with a number of thrombocytopathies are given.

\section{KEYWORDS}

Platelets, rare inherited hematologic disorders, thrombocytopathy, Glanzmann thrombasthenia, Bernard-Soulier syndrome, gray platelet syndrome

\section{INTRODUCTION}

Platelets - the smallest blood cells (2-4 microns), which are involved in homeostasis, inflammation, tissue remodeling and wound healing. They will form in the red bone marrow by lacing the site cytoplasm with a membrane from megakaryocytic. Platelets do not have a nucleus, but contain a complex system organelle. These include secretory granules, liposome's, microtubules, microfilaments, mitochondria. Also inside the platelet there is an open canalicular tubular system, penetrating right through it. There are 3 types of secretory granules, which differ in quantity, content and functions.

Thrombocytopathy - disorders of hemostasis, due to the qualitative inferiority of blood platelets with a normal number of them. 
Distinguish between hereditary and acquired. Among the primary hereditary platelet dysfunctions, the most common are atrombia, thrombocytopathy with a defect in the release of thrombocytes thrombastenia. Secondary hereditary thrombocytopathies are typical of Willebrand's disease of albinism afribinogenemia (Hsizmansky-Pudlak syndrome) of hyperelastic skin syndromes (Ehlers-Danlos), Marfan and other connective tissue dysplasias of many metabolic anomalies. Acquired thrombocytopathies with or without hemorrhagic syndrome are characteristic of many blood diseases (leukemia, hypoplastic and megaloblastic anemia) of uremia of DICsyndrome of immunopathological diseases (hemorrhagic vasculitis, lupus erythematosus, diffuse glomerulonephritis, etc.) of radiation sickness of medicinal disease when taking salicylates, xanthines, carbenicillin neurocirculatory dysfunction.

Thrombocytopathies accompanied by thrombocytopenia 1. Small size of platelets SVO, X-linked thrombocytopenia. 2. Normal size - congenital amegakaryocytic thrombocytopenia, TAP syndrome, amegakaryocytic thrombocytopenia with congenital radioulnar synostosis, autosomal dominant thrombocytopenia, familial thrombocytopathy with pre- predisposition to the development of acute myeloid leukemia. 3. Large platelets - Bernard's syndromes - Soulier, Di Giorgi, platelet type disease Willebrand, CCT, ARS syndrome, group of syndromes $\mathrm{MYH}$, $\mathrm{X}$ linked thrombocytopenia with thalassemia, Paris-Trousseau syndrome, Mediterranean macrocytopathic thrombocytopenia, dyserythropoietic anemia with thrombocytopenia.

Platelets are an important component of the system topics of hemostasis: adhesion of platelets to the site of damage vascular expectations, aggregation, secretion of coagulation factors vania, subsequent retraction of the clot, spasm of small vessels and the formation of white platelet thrombosis ba stop bleeding in the microcirculator- vessels with a diameter of up to $100 \mathrm{~nm}$. Activation super-melting system induces the formation of fibrin on the surface of activated platelets and for- mating a full blood clot. Upon activation platelets by natural stimulants such like thrombin or collagen, which are exposed when damaged waiting for the vascular wall, they are able to eject the contents of their granules containing factors tyvaniya, peroxidase, serotonin, calcium ions - Ca2 +, ADP, von Willebrand factor, platelet fibrinogen, platelet growth factor, etc. platelet count with the highest degree of activation becomes procoagulant due to exposure phosphatidyl serine and stimulates the formation thrombus. Also platelets play an essential role in the healing and regeneration of damaged tissues, by releasing growth factors that stimulate division and proliferation of cells in the damaged area. Hereditary platelet dysfunction cover a heterogeneous group of hemorrhagic diseases pain caused by congenital defects in mor- phology and / or function of platelets in normal their number. Various structures and disrupted various processes in the thrombocytes: membrane receptors, intraplatelet signaling, granules, etc. This leads to a different personal clinical manifestations of bleeding [2-4]. Thrombocytopathies are characterized primarily by the development of spontaneous and post-traumatic skin mucous bleeding. Recognition and differentiation thrombocytopathy cytopathy is based on the detection bleeding microcirculatory type with impairment functional properties, morphology and biochemistry the characteristics of platelets. Based these manifestations are based on the modern classics classification of 
thrombo-cytopathies, which is divided into 2 large groups - hereditary and acquired

Bernard-Soulier syndrome - hereditary thrombocytopathy, due to a genetic defect or a decrease in the functional activity of the complex ca GPIb-IX-V platelets. This complex is von Willebrand factor receptor, and is also necessary for fixing thrombin on the surface of platelets. From a functional point of view, adhesion is impaired platelets to the vascular subendothelial mat- Rix, which is typical for von Willebrand disease. Os- the new diagnostic criterion for this pathology serve as macrothrombocytopenia and lack of factor Von Willebrand-dependent aggregation with ristocetin, with normal amounts and normal activity the factor itself. There may also be a decrease aggregation with thrombin against the background of normal aggregation with other agonists. Deficiency of surface complex of glycoproteins GPIb-IX-V can be subsolidified by flow cytometry and genetic analysis of genes GPIBA, GPIBB and GP9. Syndrome Bernard-Soulier shows a significant bleeding microcirculatory and mixed th type, which manifests itself immediately after birth. Inheritance is autosomal recessive.

Hereditary thrombocytopathies are hereditary a therogenic group of genetically determined diseases with functional platelet deficiency due to defects in the membrane structure of cells, impaired retention of intracellular granules and damage to signaling ways of transmission. Lifethreatening bleeding with inherited platelet dysfunction are rare (1: 1000 population), while spontaneous and post-traumatic bleeding from mucous membranes, ecchymosis is common wide enough. Differential diagnosis of different personal forms of thrombocytopathy is based on the definition functional properties, morphology and biochemical characteristics thrombocyte therapy.

The syndromes of deficiency of the storage pool. These include the Hermanski-Pudlak and Chediak-Higashi syndromes. They are inherited in an autosomal recessive way. In these syndromes, there is albinism, frequent infections, pulmonary fibrosis, granulomatous colitis, prolonged bleeding time and a slight violation of blood clotting. The cause of the disease is a shortage of the contents of dense granules and/or themselves. Studies of platelet function reveal a violation of aggregation in reaction with $A D P$, epinephrine, ristocetin and collagen. At Syndrome of Chediak-Higashi dense granules, defines- electron microscopy samples are larger than normal ones and are similar in size to granules of melanocytes, white blood cells, and fibroblasts. Scott Syndrome. Thrombocytopathy, inherited by autosomal recessive type, due to the defection of phosphatidylserine output during platelet activation and, as a result, a violation of the interaction of platelets with plasma clotting factors. In this case, incomplete - valuable complexes of coagulation factors $\mathrm{Va}-\mathrm{X}$ and VIII-IXa are formed on the membrane. Defects in the binding of these complexes lead to incomplete activation of factor $X$ and prothrombion, as well as to violations of platelet factor 3 activity . A diagnostic algorithm Differential diagnosis of thrombocytopathies is extremely difficult. Very often, thrombocytopathies are masked by nosebleeds, hemorrhages, and other bleeding of the mucous membranes. The first stage of diagnosis is a sub- Robo collection of anamnesis. It is mandatory to compile a pedigree with a thorough collection of information about minimal bleeding in relatives. Important questions are: the first episode of bleeding, the presence of bleeding during teething/ change or extraction of teeth; 
whether Toni- lactone, whether there were complications in the form of long bleeding; bleeding gums when brushing zu- CWA, the presence of nasal bleeding, and if so, when Yes there are/frequency/duration; volume menstru- ation in girls of pubertal age; whether surgical intervention, whether hemorragicacal complications? If there are clinical signs of thrombocytopathies, the second stage of diagnosis is a General blood test. In many thrombocytopathies, the indicators may remain within the normal range. However, when if the platelet size changes, the automatic analyzer may not record the actual number of platelets, so it is important to count them manually in a Romanovsky-Giemsa smear. Morpho- logical analysis of platelets allows to additional information about the number and size of platelets, the presence of conglomerates and other special- of the: lack of a-granules in a large gray trom- bozita indicates that the disease grey platelet at the inclusions in the leukocyte - dependent diseases a mutation of the gene $\mathrm{MYH}$, abnormal morphology red Titov may indicate diseases associated with a mutation of the GATA-1 gene. If platelet conglomerates are detected in the smear, it is necessary to conduct a differential diagnosis with a blood sampling defect. Pseudothrombocytopenia may be the result of platelet bonding in a test tube with ethylene diamine tetraacetate. This can be easily confirmed by repeated blood sampling in a test tube with a zither.

The spread of primary hereditary thrombocytopathies in children is not established, but it is undoubtedly the most frequent genetically determined pathology of the hemostasis system. In most cases of socalled family bleeding of an unknown genesis, hereditary thrombocytopathy can be diagnosed. Their frequency in the population reaches $5 \%$.
Today, aggregometric features of a number of thrombocytopathies (table). Lack of aggregation with all agonists except ristocetin, indicates Glanzman's thrombasthenia. This diagnosis can be confirmed by the flow cytofluorimetry - quantitative assessment of the receptor platelet membranes IIb / IIla. Significantly reduced response to all concentrations of ADP indicates the presence defect of ADP-receptors P2Y12. Lowering the second aggregation waves under the influence of ADP and epinephrine and decreased aggregation with collagen may indicate destorage pool deficit. In order to confirm the deficit dense granules, it is necessary to conduct a study of the the rational consistency of dense granules by the accurate cytofluorometry and electron microscopy. Thus, recognition and differential diagnosis of thrombocytopathies should be based on study on a comprehensive study of hemostasis, study analysis of platelet morphology using light and electron microscopy, assessment of functional activity by flow cytometry, as well as carrying out genetic analysis to identify elimination of mutations correlating with different types of thrombocytopathies.

\section{REFERENCES}

1. Mansour W., Einav Y., Hauschner H. An allb mutation in patients with Glanzmann thrombasthenia located in the $\mathrm{N}$-terminus of blade 1 of the betapropeller (Asn2Asp) disrupts a calcium binding site in blade $6 . \mathrm{J}$ Thromb Haemost 2011;

2. Raccuglia G. Grey platelet syndrome:a variety of qualitative platelet disorder. Am J Med 1971.

3. Gerrard J. M., Phillips D. R., Rao G. H.. Biochemical studies of two patients with the grey platelet syndrome. J Clin Invest 1980; 
4. Levy-Toledano S., Caen J. P.,Breton-Gorius J. et al. Gray platelet syndrome: alphagranule deficiency. Its influence on platelet function. J Lab Clin Med 1981;

5. Nurden A. T., Kunicki T. J., Dupuis G.et al. Specific protein and glycoprotein deficiencies in platelets isolated from two patients with the grey platelet syndrome. Blood 1982; 